\title{
Widely Linear Adaptive Frequency Estimation of Unbalanced Three-Phase Power Systems
}

\author{
Yili Xia, Student Member, IEEE, and Danilo P. Mandic, Senior Member, IEEE
}

\begin{abstract}
A novel technique for online estimation of the fundamental frequency of unbalanced three-phase power systems is proposed. Based on Clarke's transformation and widely linear complex domain modeling, the proposed method makes use of the full second-order information within three-phase signals, thus promising enhanced and robust frequency estimation. The structure, mathematical formulation, and theoretical stability and statistical performance analysis of the proposed technique illustrate that, in contrast to conventional linear adaptive estimators, the proposed method is well matched to unbalanced system conditions and also provides unbiased frequency estimation. The proposed method is also less sensitive to the variations of the three-phase voltage amplitudes over time and in the presence of higher order harmonics. Simulations on both synthetic and real-world unbalanced power systems support the analysis.
\end{abstract}

Index Terms-Augmented complex least mean square (CLMS) (ACLMS), complex noncircularity, frequency estimation, unbalanced three-phase voltage, widely linear modeling.

\section{INTRODUCTION}

$\mathbf{I}$ $\mathrm{N}$ A power system, large dynamics of frequency oscillations can trigger a breakdown of standard phasor-based frequency estimation techniques. Since the variations from a nominal value can indicate unexpected abnormal system conditions and disturbances, fast and accurate frequency estimation in the presence of harmonics, noise, and unbalanced voltages has attracted much attention. A variety of techniques and algorithms have been developed to estimate frequency, for example, the modified zero-crossing technique [1], [2], phase-locked loop [3]-[5], least square error-based adaptive filters [6]-[8], and recursive state estimation-based nonlinear observers and extended Kalman filters [9]-[11], among which recursive state estimation-based methods have been shown to achieve accurate frequency estimation in critical cases under unbalanced voltage conditions. However, most of these methods, being based on the measurement of a single phase of the system, are limited in terms of the characterization of system frequency. In a threephase system, if line-to-line voltages are also considered, no single-phase signal adequately characterizes system frequency because six different single-phase voltage signals may exist [12]. Therefore, an optimal solution would be based on a

Manuscript received December 30, 2010; revised March 22, 2011; accepted May 8, 2011. Date of publication July 7, 2011; date of current version December 8, 2011. The Associate Editor coordinating the review process for this paper was Prof. Alessandro Ferrero.

The authors are with the Department of Electrical and Electronic Engineering, Imperial College London, SW7 2AZ London, U.K. (e-mail: yili.xia06@imperial.ac.uk; d.mandic@imperial.ac.uk).

Color versions of one or more of the figures in this paper are available online at http://ieeexplore.iee.org.

Digital Object Identifier 10.1109/TIM.2011.2159409 framework which simultaneously considers all the three-phase voltages; this would enable a unified estimation of system frequency as a whole and provide enhanced robustness.

To this end, Clarke's $\alpha \beta$ transformation has been introduced with the aim to construct a complex-valued signal with the information provided by all the three-phase voltages in a simultaneous way [13], thus equipping the classical singlephase methods with more robustness in characterizing system frequency. Based on this transformation, a number of solutions have been developed in the complex domain $\mathbb{C}$ [14]-[19]. Among them, adaptive algorithms based on the minimization of the mean square error are widely used owing to their simplicity, computational efficiency, and robust performance on frequency estimation in the presence of noise and harmonic distortions. However, in critical cases under unbalanced voltage conditions, such as when different amplitudes within the threephase source voltage or a voltage sag in one or two phases is taking place, standard phase angle calculation techniques used in a linear adaptive filter have proven suboptimal, resulting in an unavoidable oscillatory estimation error. This problem has been discussed in [20], where the complex-valued signal obtained from an unbalanced three-phase voltage source was represented as an orthogonal sum of the positive and negative sequences. Since the standard complex linear adaptive filter can only account for the positive sequences, the negative sequences introduce inevitable estimation error oscillating at twice the system frequency; recent attempts to estimate frequency under unbalanced conditions using the information extracted by all the three-phase voltages can be found in [21]-[23].

In this paper, the issue of frequency estimation using adaptive filters under unbalanced conditions is addressed based on the widely linear modeling of the complex-valued signal, derived from three-phase voltages by the $\alpha \beta$ transformation. Using recent advances in augmented complex-valued second-order statistics, we illustrate that, under unbalanced conditions, the complex-valued signal is second order noncircular (improper), for which the probability density function is not rotation invariant. For the modeling of noncircular signals, the standard linear estimation, which is based on the covariance matrix of a complex-valued random vector $\mathbf{x}$, i.e., $\mathbf{C}_{\mathbf{x x}}=E\left[\mathbf{x x}^{H}\right]$, is not adequate, and the pseudocovariance matrix $\mathbf{P}_{\mathbf{x x}}=E\left[\mathbf{x x}^{T}\right]$ should also be taken into account to describe the complete second-order behavior [24], [25]. In practice, this is achieved by virtue of widely linear modeling [24], [26], where both $\mathrm{x}$ and its complex conjugate $\mathbf{x}^{*}$ are combined into the augmented input $\mathbf{x}_{a}=\left[\mathbf{x}^{T}, \mathbf{x}^{H}\right]^{T}$. Therefore, to deal with online frequency estimation of noncircular signals, we here propose to use a widely linear modeling-based adaptive filtering method. 
This paper is organized as follows. In Section II, an overview of widely linear estimation and second-order augmented complex statistics is provided. In Section III, the noncircular nature of the complex-valued signal generated from the $\alpha \beta$ transformation of unbalanced three-phase voltages is illustrated, and a robust frequency calculation method is derived based on widely linear modeling. In Section IV, to illustrate the suitability of the proposed method, the augmented complex least mean square (CLMS) (ACLMS) algorithm [27] is used, and its superiority over the standard CLMS algorithm [16] is illustrated through analysis and via simulations on various unbalanced conditions and also in the presence of different levels of noise and higher order harmonics. Finally, Section V concludes this paper.

\section{Preliminaries}

\section{A. Widely Linear Modeling}

Consider a real-valued conditional mean estimator $\hat{y}=$ $E[y \mid x]$, which estimates the signal $y$ based on the observation $x$. For zero mean, jointly normal $y$ and $x$, the optimal solution is the linear model, $\hat{y}=\mathbf{x}^{T} \mathbf{h}$, where $\mathbf{h}=\left[h_{1}, \ldots, h_{L}\right]^{T}$ is a vector of fixed filter coefficients, $\mathbf{x}=\left[x_{1}, \ldots, x_{L}\right]^{T}$ is the regressor vector, and $(\cdot)^{T}$ is the vector transpose operator. In the complex domain, it is normally assumed that we can use the same form of estimator, leading to the standard complex linear minimum mean square error estimator

$$
\hat{y}=\hat{y}_{r}+\jmath \hat{y}_{i}=\mathbf{x}^{T} \mathbf{h}
$$

where $\jmath=\sqrt{-1}$ and subscripts $r$ and $i$ denote the real and imaginary parts of a complex variable, respectively. Note, however, that both the real and imaginary parts of complex variables are real valued, i.e., $y_{r}, y_{i} \in \mathbb{R}$; hence

$$
\hat{y}_{r}=E\left[y_{r} \mid x_{r}, x_{i}\right], \quad \hat{y}_{i}=E\left[y_{i} \mid x_{r}, x_{i}\right]
$$

and a more general form of (1) can be expressed as

$$
\hat{y}=E\left[y_{r} \mid x_{r}, x_{i}\right]+\jmath E\left[y_{i} \mid x_{r}, x_{i}\right] .
$$

Using $x_{r}=\left(x+x^{*}\right) / 2$ and $x_{i}=\left(x-x^{*}\right) / 2 \jmath$, we arrive at

$$
\hat{y}=E\left[y_{r} \mid x, x^{*}\right]+\jmath E\left[y_{i} \mid x, x^{*}\right]=E\left[y \mid \mathbf{x}, \mathbf{x}^{*}\right]
$$

that is, for an optimal linear estimator of the generality of complex signals, the "augmented" input $\left[\mathbf{x}^{T}, \mathbf{x}^{H}\right]^{T}$ must be used, leading to the widely linear estimator for complex valued data [24]

$$
\hat{y}=\mathbf{h}^{T} \mathbf{x}+\mathbf{g}^{T} \mathbf{x}^{*}=\mathbf{x}^{T} \mathbf{h}+\mathbf{x}^{H} \mathbf{g}
$$

where $\mathbf{h}$ and $\mathbf{g}$ are complex-valued coefficient vectors. The widely linear model naturally collapses into the strictly linear model $(\mathrm{g}=\mathbf{0})$ for proper data.

\section{B. Augmented Complex Statistics}

In practice, the widely linear estimate in (5) is based on a regressor vector produced by concatenating the input vector $b f x$ with its conjugate $\mathbf{x}^{*}$, to give an augmented $2 L \times 1$ input vector
$\mathbf{x}^{a}=\left[\mathbf{x}^{T}, \mathbf{x}^{H}\right]^{T}$, together with the corresponding augmented coefficient vector $\mathbf{w}^{a}=\left[\mathbf{h}^{T}, \mathbf{g}^{T}\right]^{T}$. The $2 L \times 2 L$ augmented covariance matrix [25] then becomes

$$
\mathbf{C}_{\mathbf{x x}}^{a}=E\left[\begin{array}{c}
\mathbf{x} \\
\mathbf{x}^{*}
\end{array}\right]\left[\mathbf{x}^{H} \mathbf{x}^{T}\right]=\left[\begin{array}{ll}
\mathbf{C}_{\mathbf{x x}} & \mathbf{P}_{\mathbf{x x}} \\
\mathbf{P}_{\mathbf{x x}}^{*} & \mathbf{C}_{\mathbf{x x}}^{*}
\end{array}\right]
$$

and contains the full second-order statistical information. From (6), it is clear that the covariance matrix $\mathbf{C}_{\mathbf{x x}}=E\left[\mathbf{x x}^{H}\right]$ alone does not have sufficient degrees of freedom to describe full second-order statistics, and in order to make use of all the available second-order information, we also need to consider the pseudocovariance matrix $\mathbf{P}_{\mathbf{x x}}=E\left[\mathbf{x x}^{T}\right]$. Processes with the vanishing pseudocovariance $\mathbf{P}_{\mathbf{x x}}=\mathbf{0}$ are termed second order circular (or proper); however, at present, in most realworld applications, complex signals are considered second order noncircular or improper.

\section{Frequency Estimation BASED ON Widely LINEAR ADAPTIVE FILTERING}

The voltages in the power system in a noise-free environment can be represented in a discrete time form as

$$
\begin{aligned}
& v_{a}(k)=V_{a}(k) \cos (\omega k \triangle \mathrm{T}+\phi) \\
& v_{b}(k)=V_{b}(k) \cos \left(\omega k \triangle \mathrm{T}+\phi-\frac{2 \pi}{3}\right) \\
& v_{c}(k)=V_{c}(k) \cos \left(\omega k \triangle \mathrm{T}+\phi+\frac{2 \pi}{3}\right)
\end{aligned}
$$

where $V_{a}(k), V_{b}(k)$, and $V_{c}(k)$ are the peak values of each fundamental voltage component at time instant $k, \triangle \mathrm{T}$ is the sampling interval, $\phi$ is the phase of the fundamental component, and $\omega=2 \pi f$ is the angular frequency of the voltage signal, with $f$ being the system frequency. The time-dependent threephase voltage is conveniently transformed by the orthogonal $\alpha \beta 0$ transformation matrix [13] into a zero-sequence $v_{0}$ and direct- and quadrature-axis components, $v_{\alpha}$ and $v_{\beta}$, as Clarke's transform

$$
\left[\begin{array}{l}
v_{0}(k) \\
v_{\alpha}(k) \\
v_{\beta}(k)
\end{array}\right]=\sqrt{\frac{2}{3}}\left[\begin{array}{ccc}
\frac{\sqrt{2}}{2} & \frac{\sqrt{2}}{2} & \frac{\sqrt{2}}{2} \\
1 & -\frac{1}{2} & -\frac{1}{2} \\
0 & \frac{\sqrt{3}}{2} & -\frac{\sqrt{3}}{2}
\end{array}\right]\left[\begin{array}{l}
v_{a}(k) \\
v_{b}(k) \\
v_{c}(k)
\end{array}\right] .
$$

The factor $\sqrt{2 / 3}$ is used to ensure that the system power is invariant under this transformation. When $V_{a}(k), V_{b}(k)$, and $V_{c}(k)$ are identical, $v_{0}(k)=0, v_{\alpha}(k)=A \cos (\omega k \Delta \mathrm{T}+$ $\phi)$, and $v_{\beta}(k)=A \cos (\omega k \Delta \mathrm{T}+\phi+(\pi / 2))$, with a constant amplitude $A$, where $v_{\alpha}(k)$ and $v_{\beta}(k)$ are the orthogonal coordinates of a point whose position is time variant at a rate proportional to the system frequency. In practice, normally, only the $v_{\alpha}$ and $v_{\beta}$ parts are used in the modeling [14] (known as the $\alpha \beta$ transformation), whereas the zero-sequence component $v_{0}$ is not necessary for analysis. The complex voltage signal $v(k)$ of the system, which serves as the desired signal in adaptive frequency estimation, is therefore given by

$$
v(k)=v_{\alpha}(k)+\jmath v_{\beta}(k)
$$




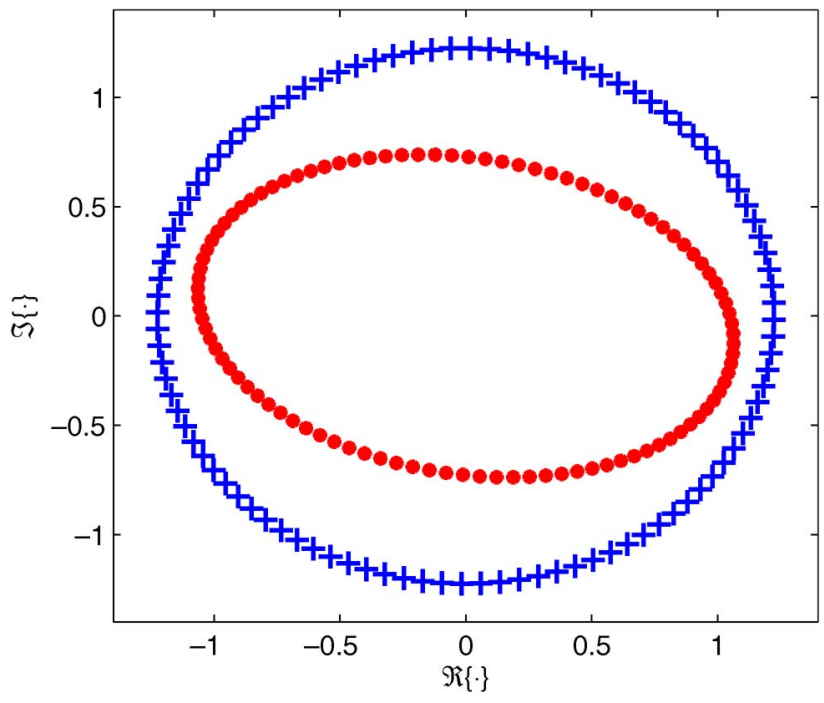

Fig. 1. Geometric view of circularity and noncircularity via a "real-imaginary" scatter plot in the complex plane. The circle denoted by "+" represents a circular complex-valued signal $v(k)$ obtained from a balanced situation where $V_{a}(k), V_{b}(k)$, and $V_{c}(k)$ are identical at 1 p.u. and time invariant. The ellipse denoted by "о" represents a noncircular complexvalued $v(k)$ obtained in an unbalanced condition with $V_{a}(k)=1$ p.u., $V_{b}(k)=0.7$ p.u., and $V_{b}(k)=0.5$ p.u.

and can be estimated iteratively as

$$
\begin{aligned}
v(k+1) & =A(k+1) e^{\jmath(\omega(k+1) \Delta \mathrm{T}+\phi)} \\
& =A e^{\jmath \omega \Delta \mathrm{T}} e^{\jmath(\omega k \Delta \mathrm{T}+\phi)}=v(k) e^{\jmath \omega \Delta \mathrm{T}}
\end{aligned}
$$

where the instantaneous system frequency $f$ is represented by the phasor $e^{\jmath \omega \Delta T}(f=(\omega / 2 \pi))$. Observe that, in normal operating conditions, samples of $v(k)$ are located on a circle in the complex plane with a constant radius $A$, depicted by " + " in Fig. 1. For a constant sampling frequency, the probability density function of $v(k)$ is rotation invariant since $v$ and $v e^{j \theta}$ have the same distribution for any real $\theta$. This, in turn, means that $v(k)$ is second order circular [28], and in this case, the frequency estimation can be performed adequately by a standard linear adaptive filter, based on the strictly linear model in (1).

The CLMS algorithm [29], which is widely used in signal processing applications, has also been used for frequency estimation of three-phase voltages [16] and can be summarized as

$$
\begin{aligned}
\hat{v}(k+1) & =v(k) w(k) \\
e(k) & =v(k+1)-\hat{v}(k+1) \\
w(k+1) & =w(k)+\mu e(k) v^{*}(k)
\end{aligned}
$$

where $w(k)$ is the weight coefficient at time instant $k, \hat{v}(k+1)$ is the estimate of desired signal $v(k+1), e(k)$ is the estimation error, and $\mu$ is the step size. Comparing (10) and the linear estimation model in (11), the system frequency can be estimated from

$$
\hat{f}(k)=\frac{1}{2 \pi \Delta \mathrm{T}} \sin ^{-1}(\Im(w(k))) .
$$

However, when the three-phase power system deviates from its normal condition, such as when the three channel voltages exhibit different levels of dips or transients, voltages $V_{a}(k)$, $V_{b}(k)$, and $V_{c}(k)$ are not identical, and samples of $v(k)$ are not allocated on a circle with a constant radius, as illustrated by the ellipse denoted by "o" in Fig. 1, causing the iterative model of $v(k)$ stated in (10) to break down. In this case, the distribution of $v(k)$ is rotation dependent (noncircular), and the signal is accurately expressed (see Appendix A for the derivation) only by using the widely linear model in (5), i.e.,

$$
v(k)=A(k) e^{\jmath(\omega k \Delta \mathrm{T}+\phi)}+B(k) e^{-\jmath(\omega k \Delta \mathrm{T}+\phi)} .
$$

In other words, when $V_{a}(k), V_{b}(k)$, and $V_{c}(k)$ are not identical, $A(k)$ is no longer a constant, and $B(k) \neq 0$, introducing a rotation-variant probability density function, implying that, in unbalanced conditions, $v(k)$ exhibits a certain degree of noncircularity. Since the widely linear model in (5) is the only secondorder optimal estimator for improper data, both $v(k)$ and its complex conjugate $v^{*}(k)$ should be considered in the frequency estimation in unbalanced cases, i.e.,

$$
\hat{v}(k+1)=\underbrace{v(k) h(k)}_{\text {standard update }}+\underbrace{v^{*}(k) g(k)}_{\text {conjugate update }}
$$

where $h(k)$ and $g(k)$ are the filter weight coefficients corresponding to the standard and conjugate updates at time instant $k$, respectively, and the estimation error $e(k)$ and the cost function $\mathcal{J}(k)$ can be defined as

$e(k)=v(k+1)-\hat{v}(k+1)$ and $\mathcal{J}(k)=|e(k)|^{2}=e(k) e^{*}(k)$.

The update of both the standard and the conjugate term weight coefficient can be obtained by using the steepest descent gradient as

$$
\begin{aligned}
& h(k+1)=h(k)-\mu \nabla_{h} \mathcal{J}(k) \\
& g(k+1)=g(k)-\mu \nabla_{g} \mathcal{J}(k)
\end{aligned}
$$

where the gradients (see [26] for more detail)

$$
\begin{aligned}
& \nabla_{h} \mathcal{J}(k)=\frac{\partial \mathcal{J}(k)}{\partial h^{*}(k)}=e(k) \frac{\partial e^{*}(k)}{\partial h^{*}(k)}+e^{*}(k) \frac{\partial e(k)}{\partial h^{*}(k)} \\
& \nabla_{g} \mathcal{J}(k)=\frac{\partial \mathcal{J}(k)}{\partial g^{*}(k)}=e(k) \frac{\partial e^{*}(k)}{\partial g^{*}(k)}+e^{*}(k) \frac{\partial e(k)}{\partial g^{*}(k)} .
\end{aligned}
$$

Since

$$
e^{*}(k)=v^{*}(k+1)-v^{*}(k) h^{*}(k)-v(k) g^{*}(k)
$$

and $\partial e(k) / \partial h^{*}(k)=\partial e(k) / \partial g^{*}(k)=0$, we obtain

$$
\begin{aligned}
& \nabla_{h} \mathcal{J}(k)=-e(k) v^{*}(k) \\
& \nabla_{g} \mathcal{J}(k)=-e(k) v(k)
\end{aligned}
$$

giving the updates of the standard and conjugate weight coefficients $h(k)$ and $g(k)$ in the form

$$
\begin{aligned}
& h(k+1)=h(k)+\mu e(k) v^{*}(k) \\
& g(k+1)=g(k)+\mu e(k) v^{*}(k) .
\end{aligned}
$$


Equations (23) and (24) describe the so-called ACLMS algorithm [27], which is designed for training widely linear adaptive filters. The stability of such a closed-loop adaptive system based on ACLMS deserves more insights and is given in Appendix B. To introduce the corresponding ACLMS-based frequency estimation method for the three-phase unbalance system, by substituting (13) into (14), the estimate $\hat{v}(k+1)$ can be expressed as

$$
\begin{aligned}
\hat{v}(k+1)= & A(k) h(k) e^{\jmath(\omega k \Delta \mathrm{T}+\phi)}+B(k) h(k) e^{-\jmath(\omega k \Delta \mathrm{T}+\phi)} \\
& +A^{*}(k) g(k) e^{-\jmath(\omega k \Delta \mathrm{T}+\phi)}+B^{*}(k) g(k) e^{\jmath(\omega k \Delta \mathrm{T}+\phi)} \\
= & \left(A(k) h(k)+B^{*}(k) g(k)\right) e^{\jmath(\omega k \Delta \mathrm{T}+\phi)} \\
& +\left(A^{*}(k) g(k)+B(k) h(k)\right) e^{-\jmath(\omega k \Delta \mathrm{T}+\phi)}
\end{aligned}
$$

while from (13), the expression for $v(k+1)$ can be rewritten as

$$
\begin{aligned}
v(k+1)=A(k+1) e^{\jmath \omega \Delta \mathrm{T}} e^{\jmath(\omega k \Delta \mathrm{T}+\phi)} & \\
& +B(k+1) e^{-\jmath \omega \Delta \mathrm{T}} e^{-\jmath(\omega k \Delta \mathrm{T}+\phi)} .
\end{aligned}
$$

Therefore, at the steady state, the first term on the righthand side (RHS) of (26) can be estimated approximately by its counterpart in (25); hence, the term $e^{\jmath \omega \Delta \mathrm{T}}$ containing the frequency information can be estimated as

$$
e^{\jmath \hat{\omega} \Delta \mathrm{T}}=\frac{A(k) h(k)+B^{*}(k) g(k)}{A(k+1)} .
$$

Comparing the second term on the RHS of (25) and (26), the evolution of the term $e^{-\jmath \omega \Delta \mathrm{T}}$ can be expressed as

$$
e^{-\jmath \hat{\omega} \Delta \mathrm{T}}=\frac{A^{*}(k) g(k)+B(k) h(k)}{B(k+1)} .
$$

Upon taking the complex conjugate, we obtain

$$
e^{\jmath \hat{\omega} \Delta \mathrm{T}}=\frac{A(k) g^{*}(k)+B^{*}(k) h^{*}(k)}{B^{*}(k+1)} .
$$

We here adopt the assumptions held implicitly in frequency estimation by adaptive filtering algorithms that $A(k+1) \approx$ $A(k)$ and, also, $B(k+1) \approx B(k)$, and thus, (27) and (29) can be simplified as

$$
\begin{aligned}
& e^{\jmath \hat{\omega} \Delta \mathrm{T}}=h(k)+\frac{B^{*}(k)}{A(k)} g(k) \\
& e^{\jmath \hat{\omega} \Delta \mathrm{T}}=h^{*}(k)+\frac{A(k)}{B^{*}(k)} g^{*}(k) .
\end{aligned}
$$

As shown in (41) in Appendix $\mathrm{A}$, the coefficient $A(k)$ is real valued, whereas $B(k)$ is complex valued, and thus, $\left(B^{*}(k) / A(k)\right)=(B(k) / A(k))^{*}$. Since (30) should be equal to (31), using $a(k)=(B(k) / A(k))^{*}$, we can find the form of $a(k)$ by solving the following quadratic equation with complexvalued coefficients:

$$
g(k) a^{2}(k)+\left(h(k)-h^{*}(k)\right) a(k)-g^{*}(k)=0 .
$$

The discriminant of this quadratic equation is given by

$$
\begin{aligned}
\triangle & =\sqrt{\left(h(k)-h^{*}(k)\right)^{2}+4|g(k)|^{2}} \\
& =2 \sqrt{-\Im^{2}(h(k))+|g(k)|^{2}}
\end{aligned}
$$

where the operator $\Im(\cdot)$ represents the imaginary part of a complex-valued number. Since $a(k)$ is complex valued, the discriminant is negative, and the two roots can be found as

$$
\begin{aligned}
& a_{1}(k)=\frac{-\jmath \Im(h(k))+\jmath \sqrt{\Im^{2}(h(k))-|g(k)|^{2}}}{g(k)} \\
& a_{2}(k)=\frac{-\jmath \Im(h(k))-\jmath \sqrt{\Im^{2}(h(k))-|g(k)|^{2}}}{g(k)} .
\end{aligned}
$$

From (30), the phasor $e^{\jmath \hat{\omega} \Delta \mathrm{T}}$ is estimated either by using $h(k)+a_{1}(k) g(k)$ or $h(k)+a_{2}(k) g(k)$. Since the system frequency is far smaller than the sampling frequency, the imaginary part of $e^{\jmath \hat{\omega} \Delta \mathrm{T}}$ is positive, thus excluding the solution based on $a_{2}(k)$. The system frequency $\hat{f}(k)$ is therefore estimated in the form

$$
\hat{f}(k)=\frac{1}{2 \pi \Delta \mathrm{T}} \sin ^{-1}\left(\Im\left(h(k)+a_{1}(k) g(k)\right)\right) .
$$

The aforementioned equation is a generic widely linear extension of the standard linear frequency estimation method and can be implemented by any type of widely linear adaptive filter [30], [31]. In addition, when the system is balanced, $g(k)=0$, and the estimator in (35) simplifies into the standard linear CLMS-based estimation.

\section{Simulations}

The adaptive frequency estimator in (35) based on the widely linear ACLMS algorithm was applied to estimate the fundamental frequency variations from sampled values of voltage signals across several typical power system operating conditions and was compared with the standard CLMS algorithm in (12). Simulations were performed in the Matlab programming environment with a sampling rate of $5 \mathrm{kHz}$, and the step size $\mu$ of both algorithms was set to be 0.01 in all simulations. To quantify the degree of noncircularity in different unbalanced conditions, the circularity index $\eta$ was used, given by [32]

$$
\eta=\frac{\tau_{v}^{2}}{\sigma_{v}^{2}}
$$

where $\sigma_{v}^{2}=E\left[v(k) v^{*}(k)\right]$ is the variance of $v$ and $\tau_{v}^{2}=$ $\left|E\left[v(k)^{2}\right]\right|$ is the absolute value of the pseudovariance of $v$. The values of the noncircularity index $\eta$ lie in the interval $[0,1]$, the value of 0 indicating that $v(k)$ is perfectly circular, otherwise indicating a second-order noncircular (improper) $v(k)$.

In the first set of simulations, the simulated power system was in its normal operating condition at $50 \mathrm{~Hz}$, with a balanced distortion-free three-phase input signal with unity magnitude, as shown in Fig. 2(a) (left-hand part). Both algorithms were initialized at $50.5 \mathrm{~Hz}$ and converged to $50 \mathrm{~Hz}$ in a very similar 

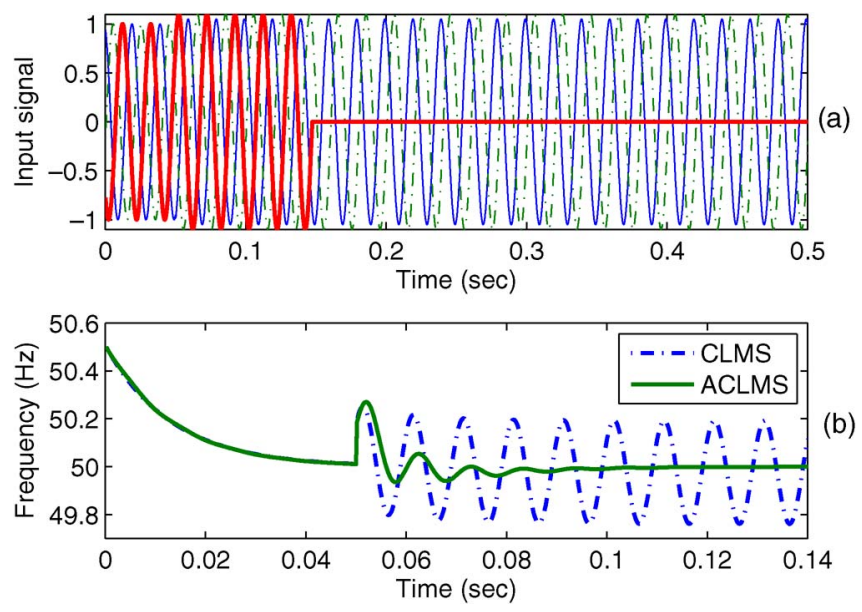

Fig. 2. Frequency estimation under unbalanced conditions. (a) To generate an unbalanced condition, an extra 0.1-p.u. magnitude was imposed on (dashed line) phase $b$ and (thick line) phase $c$, plus a 0.05-p.u. magnitude on (solid line) phase $a$, and subsequently, a 100\% single-phase voltage sag in the third channel of three-phase system occurred at $t=0.15 \mathrm{~s}$. (b) Frequency estimation by both algorithms for up to $0.15 \mathrm{~s}$. The frequency parameter of both linear and widely linear adaptive filters was initialized at $50.5 \mathrm{~Hz}$, with the true system frequency at $50 \mathrm{~Hz}$.

way, as shown in Fig. 2(b). In a balanced condition, the circularity index was $\eta=0$, indicating a perfect circular $v(k)$. Then, an extra 0.1-per-unit (p.u.) magnitude was imposed on phases $b$ and $c$, together with a 0.05 -p.u. magnitude on phase $a$, leading to an unbalanced three-phase power system and, consequently, a noncircular complex-valued input signal $v(t)$ from $t=0.05 \mathrm{~s}$, with the noncircularity index $\eta=0.0306$. There was an inevitable oscillation error at twice the system frequency of $100 \mathrm{~Hz}$ when using the linear CLMS algorithm (see right-hand part of Fig. 2(b), and the reason is given in Appendix C) due to its submodeling of the unbalanced widely linear system, whereas the capacity of the proposed method in accurately estimating (no steady-state errors) the frequency in an unbalanced situation can clearly be observed. To illustrate the statistical advantage of the ACLMS-based estimator over the CLMSbased estimator, we next performed bias and variance analysis of both algorithms in a noisy environment. According to (13) and following the approach in [33], the Cramer-Rao lower bound (CRLB), which characterizes the variance of a frequency estimator on a single tone exponential contaminated by zeromean complex-valued doubly white Gaussian noise ${ }^{1}$ with variance $\sigma_{n}^{2}$, can be expressed as (37), shown at the bottom of the page. Fig. 3(a) and (b) illustrates the statistical bias and variance performance of CLMS and ACLMS on frequency estimation of the unbalanced system against different levels of noise. The result was obtained by averaging 1000 independent trails. The

\footnotetext{
${ }^{1}$ For white Gaussian noise, $n=n_{r}+\jmath n_{i}, \sigma_{n_{r}}^{2}=\sigma_{n_{i}}^{2}=(1 / 2) \sigma_{n}^{2}$, and double whiteness implies the uncorrelated real and imaginary channels.
}

unbiased property of the ACLMS estimator can be observed in the high SNR region, whereas due to the submodeling, CLMS always performed a biased estimation. In Fig. 3(b), the ACLMS achieved a very small error variance approaching the CRLB within $4 \mathrm{~dB}$ when the SNR was between 30 and $50 \mathrm{~dB}$. It is also interesting to note that the error variance of CLMS was nearly unaffected by the noise. This is due to the fact that, compared with noise, the unavoidable estimation oscillation error experienced by CLMS [which can be observed from Fig. 2(b)] constituted the major part of the estimation variance.

In the next simulation, a $100 \%$ single-phase voltage sag suddenly occurred at $0.15 \mathrm{~s}$ in channel $c\left(V_{c}=0\right)$ of the unbalanced three-phase system with the degree of noncircularity $\eta=0.8004$; the geometric illustration of the noncircularity of $v(k)$ is shown in Fig. 4. The tracking performance in Fig. 5 shows that the CLMS algorithm lost its frequency tracking capability, whereas after convergence, the ACLMS algorithm was able to accurately track the system frequency, with no oscillations.

The second set of simulations addressed the impact of oscillatory variations of magnitude on the estimated frequency. In this case study, at $t=0.05 \mathrm{~s}$, the magnitudes of balanced three-phase voltages changed from their normal values according to $V_{a}(k)=1+0.05 \sin (2 \pi k \Delta \mathrm{T}), V_{b}(k)=1+$ $0.1 \sin (2 \pi k \Delta \mathrm{T})$, and $V_{c}(k)=1+0.15 \sin (2 \pi k \Delta \mathrm{T})$, causing a noncircular behavior of $v(k)$ with a degree of noncircularity $\eta=0.0012$. Fig. 6 shows that the tracking performance of the proposed ACLMS-trained widely linear estimator exhibited a very small oscillatory steady-state error with a maximum of $0.01 \mathrm{~Hz}$, whereas the CLMS-based estimator diverged. This small oscillatory steady-state error of the ACLMS stems from the assumptions [see the simplification of (27) and (29)] that, at two successive time instants, $A(k+1) \approx A(k)$ and $B(k+$ $1) \approx B(k)$.

The next set of simulations shows that, when the input signal is contaminated with harmonics, the estimated frequency is subject to an oscillatory steady-state error. In Fig. 7(a), a balanced $10 \%$ third harmonic and a $5 \%$ fifth harmonic of the fundamental frequency were added into the unbalanced three-phase power system at $t=0.05 \mathrm{~s}$. The ACLMS algorithm achieved better performance with a smaller oscillation error at the steady state as compared with the CLMS algorithm; this advantage was most pronounced when the magnitudes of the third and fifth harmonics varied over a range of $(0.0-0.5)$ p.u., as shown in Fig. 7(b), with the corresponding degree of noncircularity $\eta$ varying from 0.0306 to 0.0413 .

In the following set of simulations, the performances of the proposed widely linear ACLMS and the strictly linear CLMS were compared for the case of frequency variation. In Fig. 8(a), a balanced voltage signal was affected by the $2-\mathrm{Hz}$ step change in frequency from 50 to $52 \mathrm{~Hz}$ at $0.05 \mathrm{~s}$. In this case, both

$$
\operatorname{var}(\hat{f}) \geq \frac{\sigma_{n}^{2}}{2 \sum_{k=0}^{K}(2 \pi k \Delta \mathrm{T})^{2}\left((A(k)+B(k))^{2} \sin ^{2}(2 \pi f k \Delta \mathrm{T}+\phi)+(A(k)-B(k))^{2} \cos ^{2}(2 \pi f k \Delta \mathrm{T}+\phi)\right)}
$$




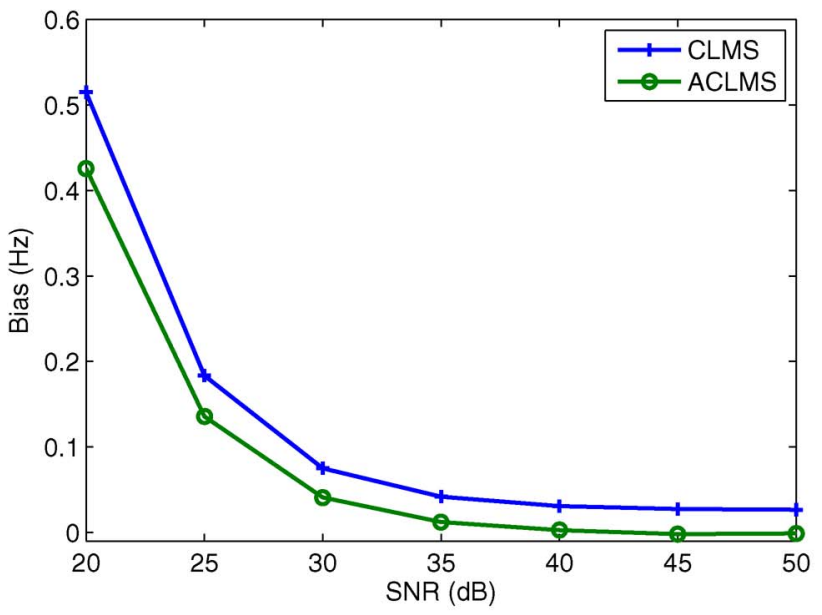

(a)

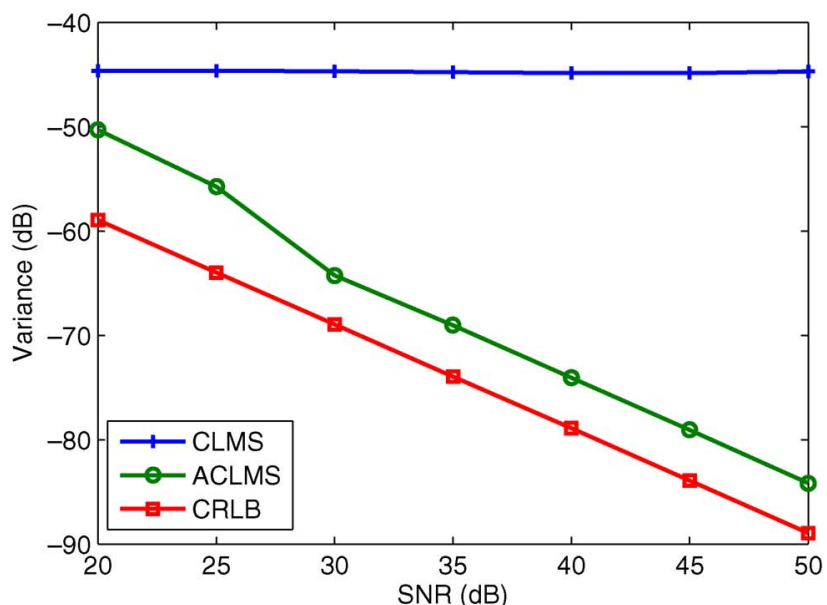

(b)

Fig. 3. Comparison of the proposed ACLMS and CLMS at different SNRs, obtained by averaging 1000 independent trials. (a) Bias error. (b) Variance.

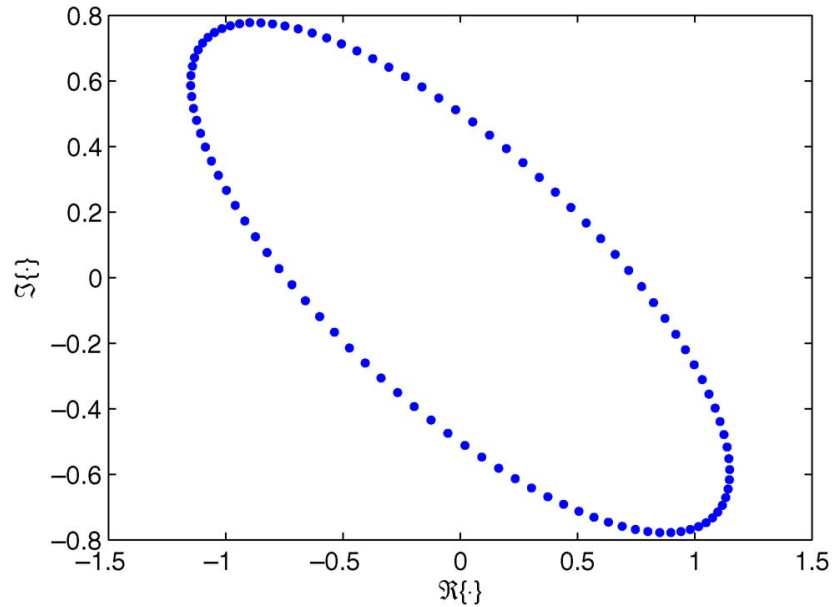

Fig. 4. Geometric view of the noncircularity of $v(k)$ via a "real-imaginary" scatter plot when a $100 \%$ single-phase voltage sag happened in phase channel $c\left(V_{c}=0\right)$.

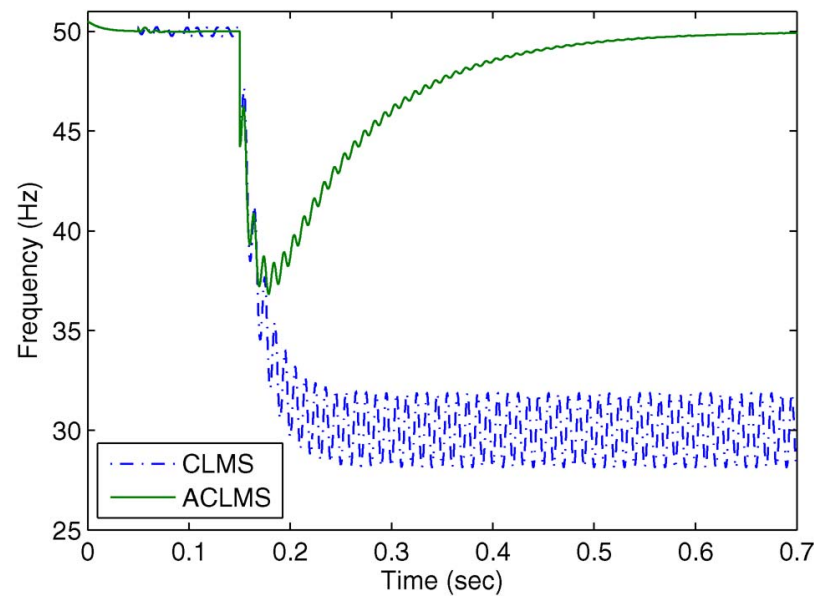

Fig. 5. Frequency estimation by the standard and widely linear algorithms for up to $0.7 \mathrm{~s}$. The voltage sag occurred at $t=0.15 \mathrm{~s}$.

algorithms achieved accurate frequency estimation; however, at $t=0.3 \mathrm{~s}$ when the system signal became unbalanced and the frequency was simultaneously changed back to $50 \mathrm{~Hz}$, the

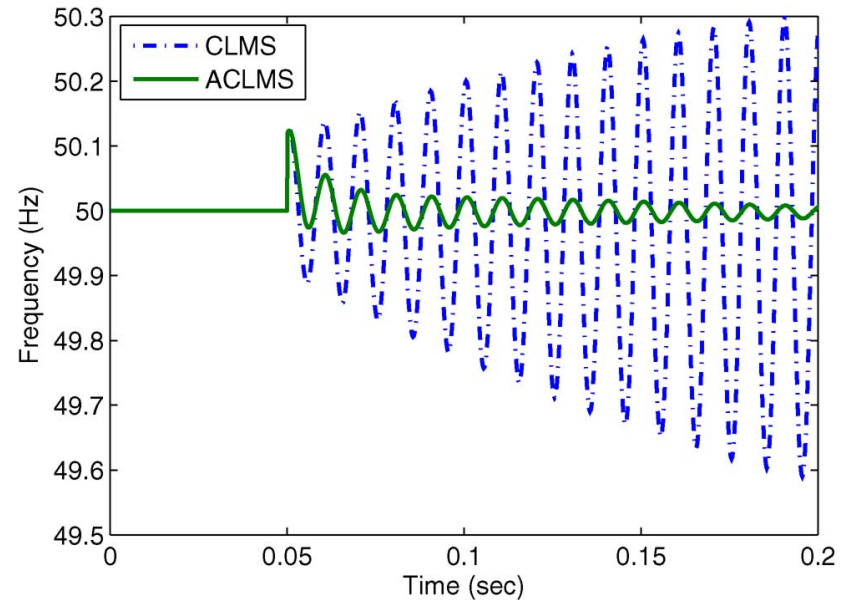

Fig. 6. Impact of oscillatory variations of amplitude on the frequency estimation by CLMS and ACLMS.

ACLMS algorithm could still track the system frequency with no steady-state error, whereas the CLMS algorithm suffered from the oscillation error. In Fig. 8(b), the 50-Hz fundamental frequency of the unbalanced signal arose and decayed at a rate of $5 \mathrm{~Hz} / \mathrm{s}$; the estimated frequency obtained by the ACLMS algorithm followed the actual system frequency very closely with a delay of $0.05 \mathrm{~s}$.

In the last set of simulations, we considered a real-world problem, where unbalanced three-phase voltages were recorded at a 110/20/10 kV transformer station. The REL 531 numerical line distance protection terminal, produced by ABB Ltd., was installed in the station and was used to monitor changes in the three "phase-ground" voltages. The device was set to record whenever the phase voltage value dropped below $90 \%$ of its norminal value for longer than $20 \mathrm{~ms}$. The measured three "phase-ground" voltages with a system frequency of $50 \mathrm{~Hz}$ were sampled at $1 \mathrm{kHz}$ and were normalized with respect to their normal peak voltage value. Two case studies are provided. In the first case, as shown in Fig. 9(a), at around $t=$ $0.06 \mathrm{~s}$, phase $v_{a}$ experienced a shortcut with earth, and the voltage dropped to $44 \%$ of its normal value. Meanwhile, both phases $v_{b}$ and $v_{c}$ experienced $36 \%$ and $50 \%$ voltage swells, 


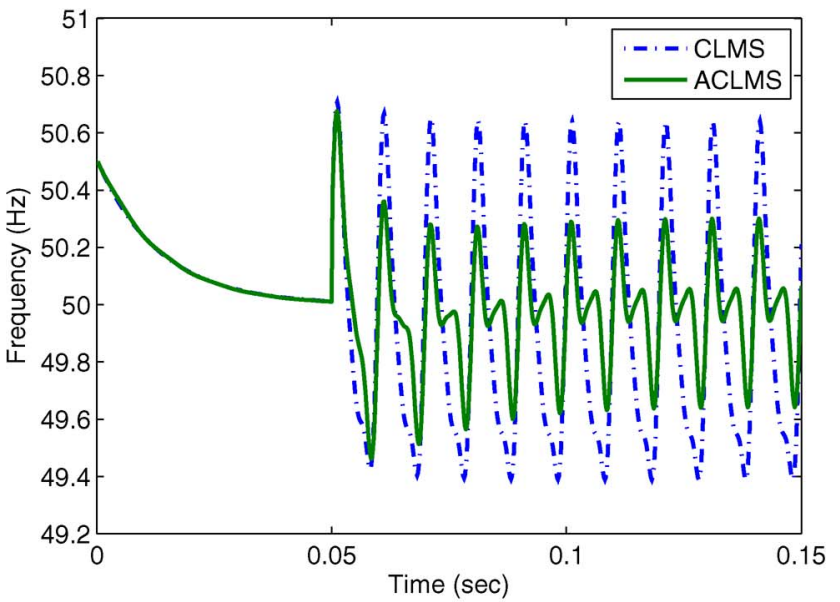

(a)

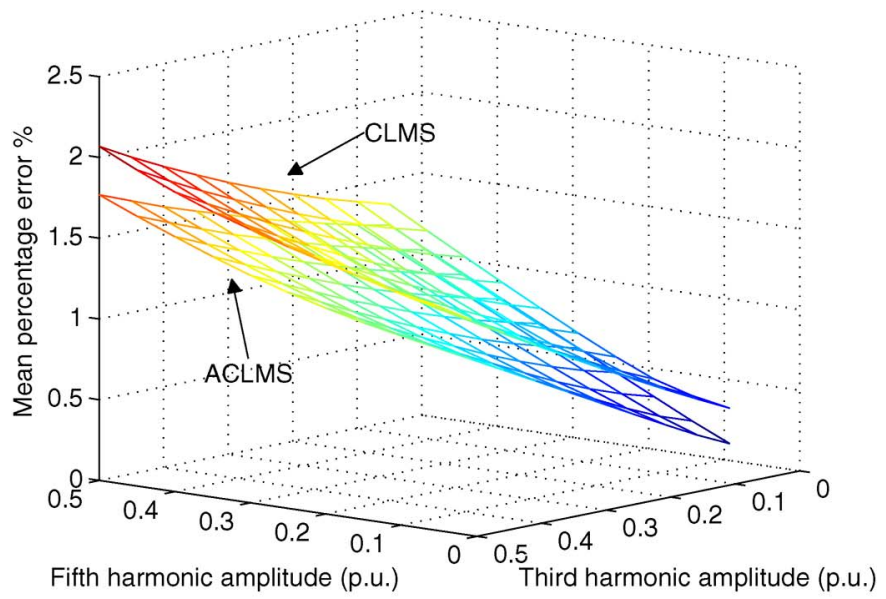

(b)

Fig. 7. Frequency estimation for phase voltages contaminated with harmonics. (a) $10 \%$ p.u. third harmonic and $5 \%$ p.u. fifth harmonic were added into the unbalanced power system at $t=0.05 \mathrm{~s}$. (b) Mean percentage error of CLMS and ACLMS algorithms over a range of amplitudes of both third harmonic and fifth harmonic at the steady state.
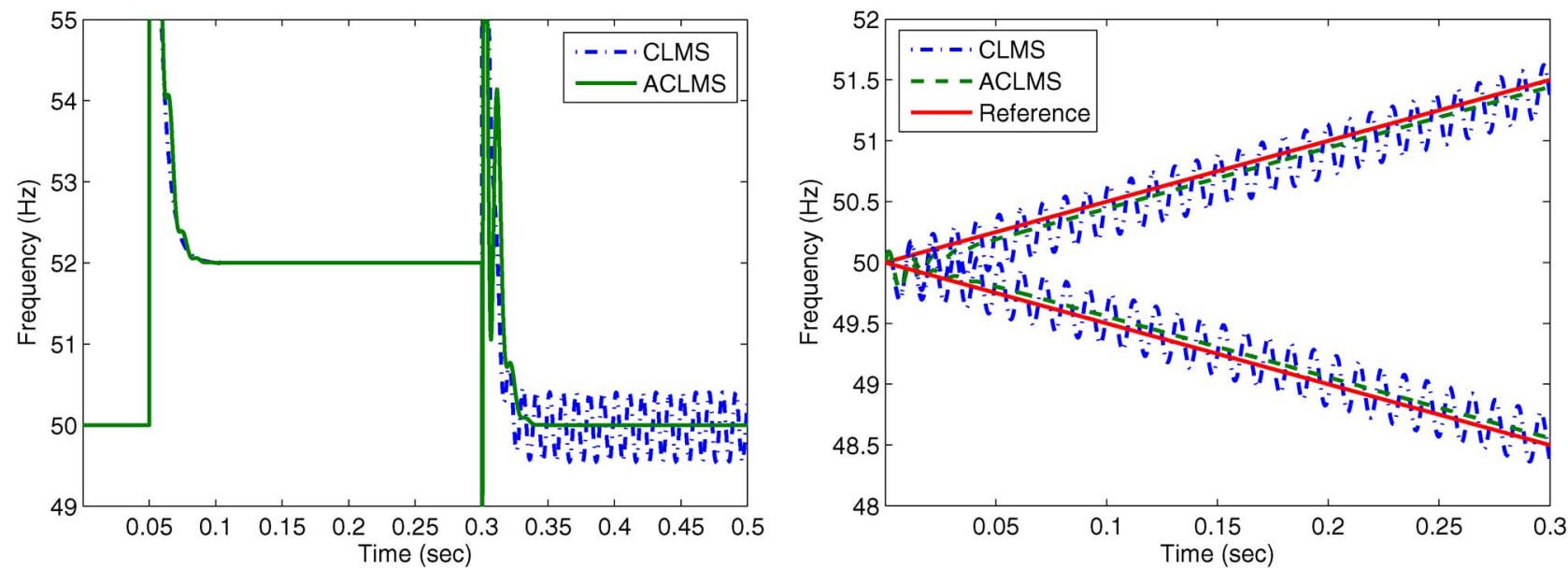

Fig. 8. Frequency estimation under frequency variations. (a) Unbalanced and noncircular system input suffered from sudden frequency changes. (b) Unbalanced and noncircular system input suffered from the frequency rise and decay at a rate of $5 \mathrm{~Hz} / \mathrm{s}$.

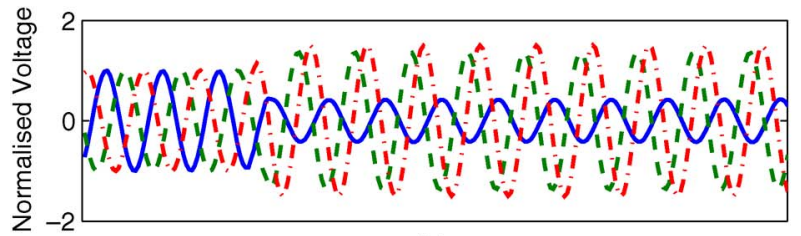

(a)

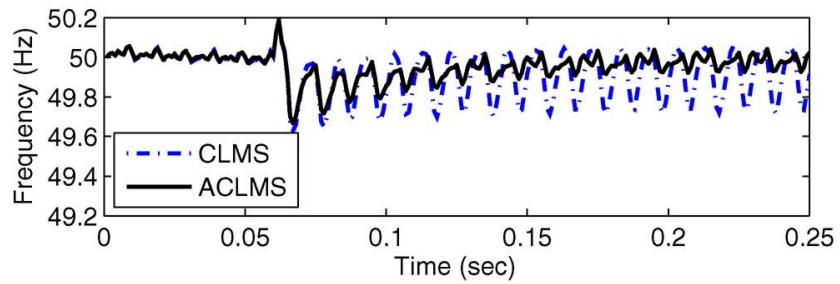

(b)

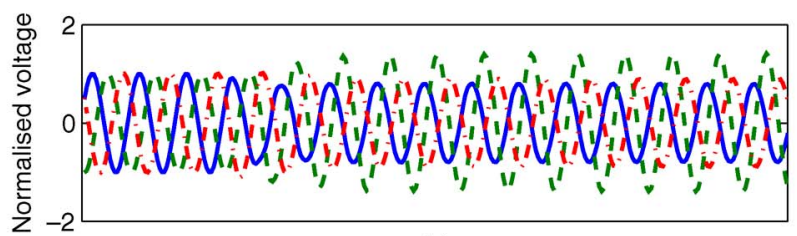

(c)

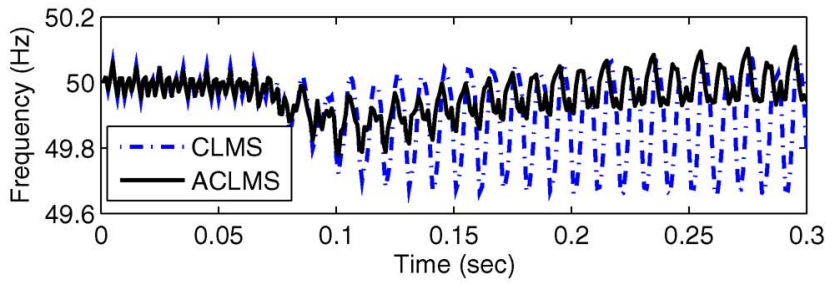

(d)

Fig. 9. Frequency estimation for real-world unbalanced three-phase voltage. (a) Time series of the real-world unbalanced three-phase voltage, where $v_{a}$ experienced a shortcut with earth. (b) Frequency estimation of both algorithms. (c) Time series of the real-world unbalanced three-phase voltage, where both $v_{a}$ and $v_{c}$ experienced shortcuts with earth. (d) Frequency estimation of both algorithms.

respectively, giving a degree of noncircularity of $\eta=0.0333$. In the second case study, as shown in Fig. 9(c), at around $t=0.07 \mathrm{~s}$, both phases $v_{a}$ and $v_{c}$ experienced shortcuts with earth, resulting in $20 \%$ and $11 \%$ voltage drops, respectively, and a $41 \%$ voltage swell in phase $v_{b}$, giving a degree of noncircularity of $\eta=0.0705$. The frequency tracking capabilities of the 
proposed ACLMS and standard CLMS methods are shown in Fig. 9(b) and (d). Both methods provided accurate responses under normal operating conditions; however, as expected, the CLMS failed to deal with unbalanced situations, whereas the fluctuations of the estimated frequency produced by ACLMS were much lower than those of the CLMS method.

\section{CONCLUSION}

We have shown that the complex-valued signal, obtained from the $\alpha \beta$ transformation of unbalanced three-phase voltages, is second order noncircular (improper), which renders strictly linear adaptive filtering-based frequency estimation methods suboptimal. To deal with frequency estimation based on noncircular signals, a widely linear adaptive filter and a corresponding robust frequency estimation method have been introduced, achieving enhanced performance under various unbalanced conditions. The proposed method has also been shown to be less sensitive to higher order harmonics and noises as compared with standard linear adaptive filters. Simulations over a range of unbalanced system conditions support the approach.

\section{APPENDIX A}

From the standard three-phase system in (7) and (8), the components $v_{\alpha}(k)$ and $v_{\beta}(k)$ of the complex voltage $v(k)=$ $v_{\alpha}(k)+\jmath v_{\beta}(k)$, obtained using the $\alpha \beta$ transformation, can be derived as

$$
\begin{aligned}
v_{\alpha}(k)= & \sqrt{\frac{2}{3}}\left(v_{a}(k)-\frac{v_{b}(k)}{2}-\frac{v_{c}(k)}{2}\right) \\
= & \sqrt{\frac{2}{3}}\left(V_{a}(k) \cos (\omega k \triangle \mathrm{T}+\phi)\right. \\
& -\frac{1}{2} V_{b}(k) \cos \left(\omega k \triangle \mathrm{T}+\phi-\frac{2 \pi}{3}\right) \\
& \left.-\frac{1}{2} V_{c}(k) \cos \left(\omega k \triangle \mathrm{T}+\phi+\frac{2 \pi}{3}\right)\right) \\
= & \sqrt{\frac{2}{3}}\left(V_{a}(k) \cos (\omega k \triangle \mathrm{T}+\phi)-\frac{V_{b}(k)}{2}\right. \\
& \times\left(\cos (\omega k \triangle \mathrm{T}+\phi) \cos \frac{2 \pi}{3}+\sin (\omega k \triangle \mathrm{T}+\phi) \sin \frac{2 \pi}{3}\right) \\
& -\frac{V_{c}(k)}{2}\left(\cos (\omega k \triangle \mathrm{T}+\phi) \cos \frac{2 \pi}{3}\right. \\
= & \left(\frac{\sqrt{6} V_{a}(k)}{3}+\frac{\sqrt{6}\left(V_{b}(k)+V_{c}(k)\right)}{12}\right) \cos (\omega k \triangle \mathrm{T}+\phi) \\
& -\frac{\sqrt{2}\left(V_{b}(k)-V_{c}(k)\right)}{4} \sin (\omega k \triangle \mathrm{T}+\phi) \\
& (38) \\
&
\end{aligned}
$$

$$
\begin{aligned}
v_{\beta}(k)= & \sqrt{\frac{2}{3}}\left(\frac{\sqrt{3} v_{b}(k)}{2}-\frac{\sqrt{3} v_{c}(k)}{2}\right) \\
= & \frac{\sqrt{2}}{2}\left(v_{b}(k)-v_{c}(k)\right) \\
= & \frac{\sqrt{2}}{2}\left(V _ { b } ( k ) \left(\cos (\omega k \triangle \mathrm{T}+\phi) \cos \frac{2 \pi}{3}\right.\right. \\
& \left.+\sin (\omega k \triangle \mathrm{T}+\phi) \sin \frac{2 \pi}{3}\right) \\
-V_{c}(k)\left(\cos (\omega k \triangle \mathrm{T}+\phi) \cos \frac{2 \pi}{3}\right. & \left.\left.-\sin (\omega k \triangle \mathrm{T}+\phi) \sin \frac{2 \pi}{3}\right)\right) \\
= & -\frac{\sqrt{2}\left(V_{b}(k)-V_{c}(k)\right)}{4} \cos (\omega k \triangle \mathrm{T}+\phi) \\
& +\frac{\sqrt{6}\left(V_{b}(k)+V_{c}(k)\right)}{4} \sin (\omega k \triangle \mathrm{T}+\phi) .
\end{aligned}
$$

Keeping in mind that

$$
\begin{aligned}
& \cos (\omega k \triangle \mathrm{T}+\phi)=\frac{e^{\jmath(\omega k \Delta \mathrm{T}+\phi)}+e^{-\jmath(\omega k \Delta \mathrm{T}+\phi)}}{2} \\
& \sin (\omega k \Delta \mathrm{T}+\phi)=\frac{e^{\jmath(\omega k \Delta \mathrm{T}+\phi)}-e^{-\jmath(\omega k \Delta \mathrm{T}+\phi)}}{2 \jmath}
\end{aligned}
$$

we have

$$
\begin{aligned}
v(k)= & v_{\alpha}(k)+\jmath v_{\beta}(k) \\
= & \left(\frac{\sqrt{6} V_{a}(k)}{3}+\frac{\sqrt{6}\left(V_{b}(k)+V_{c}(k)\right)}{12}\right) \\
& \times \frac{e^{\jmath(\omega k \Delta \mathrm{T}+\phi)}+e^{-\jmath(\omega k \Delta \mathrm{T}+\phi)}}{2} \\
& -\frac{\sqrt{2}\left(V_{b}(k)-V_{c}(k)\right)}{4} \frac{e^{\jmath(\omega k \Delta \mathrm{T}+\phi)}-e^{-\jmath(\omega k \Delta \mathrm{T}+\phi)}}{2 \jmath} \\
& -\frac{\sqrt{2}\left(V_{b}(k)-V_{c}(k)\right)}{4} \jmath \frac{e^{\jmath(\omega k \Delta \mathrm{T}+\phi)}+e^{-\jmath(\omega k \Delta \mathrm{T}+\phi)}}{2} \\
& +\frac{\sqrt{6}\left(V_{b}(k)+V_{c}(k)\right)}{4} \jmath \frac{e^{\jmath(\omega k \Delta \mathrm{T}+\phi)}-e^{-\jmath(\omega k \Delta \mathrm{T}+\phi)}}{2 \jmath} \\
= & \underbrace{\sqrt{6}\left(V_{a}(k)+V_{b}(k)+V_{c}(k)\right)}_{A(k)} e^{\jmath(\omega k \Delta \mathrm{T}+\phi)} \\
& +\underbrace{\left.\frac{\sqrt{6}\left(2 V_{a}(k)-V_{b}(k)-V_{c}(k)\right)}{\underbrace{(k)}}-\frac{\sqrt{2}\left(V_{b}(k)-V_{c}(k)\right)}{4} \jmath\right)}_{12} \\
& \times e^{-\jmath(\omega k \Delta \mathrm{T}+\phi)} .
\end{aligned}
$$


Then, the complex-valued $v(k)$ in (41) can be written in the form of a standard part (left-hand term) and a conjugate part (right-hand term). Augmented complex statistics [26], [28] shows that $v(k)$ is second order circular with a rotationinvariant probability density function in the complex plane if and only if $B(k)$ vanishes and $A(k)$ is a constant, which can only be achieved when $V_{a}(k), V_{b}(k)$, and $V_{c}(k)$ are identical at each time instant and (41) simplifies into (10). In unbalanced conditions, $A(k)$ is a real-valued variable, but $B(k) \neq 0$ and can be complex valued, which results in a rotation-variant distribution of $v(k)$ in the complex plane and a second-order noncircular $v(k)$.

\section{APPENDIX B}

This appendix outlines the theoretical stability analysis of the proposed three-phase frequency estimator using the ACLMS algorithm [27]. To achieve this, using the widely linear estimator in (5), the augmented weight vector and augmented input vector are defined as $\mathbf{w}^{a}(k)=[h(k), g(k)]^{T}$ and $\mathbf{v}^{a}(k)=$ $\left[v(k), v^{*}(k)\right]^{T}$. The widely linear estimate of the desired signal $v(k+1)$ thus becomes

$$
\begin{aligned}
v(k+1) & =v(k) h_{\mathrm{o}}+v^{*}(k) g_{\mathrm{o}} \\
& =\mathbf{v}^{a T}(k) \mathbf{w}_{\mathrm{o}}^{a}
\end{aligned}
$$

where $h_{\mathrm{o}}$ and $g_{\mathrm{o}}$ are the optimal weight coefficients for the standard and the conjugate part, respectively, the optimal augmented filter weight vector $\mathbf{w}_{\mathrm{o}}^{a}=\left[h_{\mathrm{o}}, g_{\mathrm{o}}\right]^{T}$, and the weight error vector $\widetilde{\mathbf{w}}^{a}(k)=\mathbf{w}_{\mathrm{o}}^{a}-\mathbf{w}^{a}(k)$. The evolution of the weight error vector can be analyzed based on (23) and (24) as

$$
\widetilde{\mathbf{w}}^{a}(k+1)=\widetilde{\mathbf{w}}^{a}(k)-\mu e(k) \mathbf{v}^{a *}(k)
$$

where the widely linear output error

$$
\begin{aligned}
e(k) & =v(k+1)-\hat{v}(k+1) \\
& =\mathbf{v}^{a T}(k) \widetilde{\mathbf{w}}^{a}(k) .
\end{aligned}
$$

Substituting (44) into (43) gives

$$
\widetilde{\mathbf{w}}^{a}(k+1)=\left(1-\mu \mathbf{v}^{a T}(k) \mathbf{v}^{a *}(k)\right) \widetilde{\mathbf{w}}^{a}(k) .
$$

Taking the statistical expectation of the aforementioned equation and following the standard convergence analysis, we obtain [26], [34]

$$
\left|1-2 \mu \sigma_{v}^{2}\right|<1
$$

to give

$$
0<\mu<\frac{1}{\sigma_{v}^{2}}
$$

where $\sigma_{v}^{2}$ is the variance of $v(k)$. Comparing with the convergence analysis of the CLMS algorithm given in [16], the upper bound for the step size of ACLMS is half that of CLMS. For more details on the convergence analysis of ACLMS, see [34] and [35].

\section{APPENDIX C}

This appendix gives a theoretical illustration of the suboptimality of strictly linear adaptive filters for frequency estimation of unbalanced three-phase voltage systems. The strictly linear CLMS algorithm used in standard frequency estimation is summarized in [16]. In any unbalanced condition, (13) stands, and the estimator $\hat{v}(k+1)$, obtained by using the CLMS algorithm, can be expressed as

$$
\hat{v}(k+1)=\left(A(k) e^{\jmath(\omega k \Delta \mathrm{T}+\phi)}+B(k) e^{-\jmath(\omega k \Delta \mathrm{T}+\phi)}\right) w(k) .
$$

In the steady state, $\hat{v}(k+1) \approx v(k+1)$, resulting in

$$
\begin{aligned}
& w(k) \\
& =\frac{A(k+1) e^{\jmath(\omega k \Delta \mathrm{T}+\phi)} e^{j \omega \Delta \mathrm{T}}+B(k+1) e^{-\jmath(\omega k \Delta \mathrm{T}+\phi)} e^{-j \omega \Delta \mathrm{T}}}{A(k) e^{\jmath(\omega k \Delta \mathrm{T}+\phi)}+B(k) e^{-\jmath(\omega k \Delta \mathrm{T}+\phi)}} .
\end{aligned}
$$

Under the standard assumptions that $A(k+1) \approx A(k)$ and $B(k+1) \approx B(k)$, we have

$$
w(k)=e^{-j \omega \Delta \mathrm{T}}+\frac{e^{j \omega \Delta \mathrm{T}}-e^{-j \omega \Delta \mathrm{T}}}{1+\frac{B(k)}{A(k)} e^{-2 \jmath(\omega k \Delta \mathrm{T}+\phi)}}
$$

where $(B(k) / A(k))$ is an unknown parameter and $w(k)$ is periodic as $w(k)=w(k+(1 / 2 f \Delta \mathrm{T}))$. In (12), function $\sin ^{-1}$ is a monotonic function, resulting in periodic oscillations in the estimated frequency $\hat{f}(k)$. The cycle frequency due to undermodeling is $2 f$ when using standard linear adaptive filters for frequency estimation of unbalanced power systems, whereas for balanced power systems, $B(k)=0$, and the standard linear estimate in (12) is adequate.

\section{ACKNOWLEDGMENT}

The authors would like to thank Z. Blazic of Elektroprenos, $\mathrm{BiH}$, for providing real-world data, fruitful discussions, and expert advice.

\section{REFERENCES}

[1] G. P. Hancke, "The optimal frequency estimation of a noisy sinusoidal signal," IEEE Trans. Instrum. Meas., vol. 39, no. 6, pp. 843-846, Dec. 1990.

[2] O. Vainio and S. Ovaska, "Digital filtering for robust $50 / 60 \mathrm{~Hz}$ zero-crossing detectors," IEEE Trans. Instrum. Meas., vol. 45, no. 2, pp. 426-430, Apr. 1996.

[3] V. Kaura and V. Blasko, "Operation of a phase locked loop system under distorted utility conditions," IEEE Trans. Ind. Appl., vol. 33, no. 1, pp. 58-63, Jan./Feb. 1997.

[4] S. K. Chung, "A phase tracking system for three phase utility interface inverters," IEEE Trans. Power Electron., vol. 15, no. 3, pp. 431-438, May 2000.

[5] H. Karimi, M. Karimi-Ghartemani, and M. R. Iravani, "Estimation of frequency and its rate of change for applications in power systems," IEEE Trans. Power Del., vol. 19, no. 2, pp. 472-480, Apr. 2004.

[6] M. S. Sachdev and M. M. Giray, "A least square technique for determining power system frequency," IEEE Trans. Power App. Syst., vol. PAS-104, no. 2, pp. 437-444, Feb. 1985. 
[7] I. Kamwa and R. Grondin, "Fast adaptive schemes for tracking voltage phasor and local frequency in power transmission and distribution systems," IEEE Trans. Power Del., vol. 7, no. 2, pp. 789-795, 1992.

[8] V. V. Terzija, "Improved recursive Newton-type algorithm for frequency and spectra estimation in power systems," IEEE Trans. Instrum. Meas., vol. 52, no. 5, pp. 1654-1659, Oct. 2003.

[9] F. Nagy, "Measurement of signal parameters using nonlinear observers," IEEE Trans. Instrum. Meas., vol. 41, no. 1, pp. 152-155, Feb. 1992.

[10] P. K. Dash, R. K. Jena, G. Panda, and A. Routray, "An extended complex Kalman filter for frequency measurement of distorted signals," IEEE Trans. Instrum. Meas., vol. 49, no. 4, pp. 746-753, Aug. 2000.

[11] A. Routray, A. K. Pradhan, and K. P. Rao, "A novel Kalman filter for frequency estimation of distorted signals in power systems," IEEE Trans. Instrum. Meas., vol. 51, no. 3, pp. 469-479, Jun. 2002.

[12] V. Eckhardt, P. Hippe, and G. Hosemann, "Dynamic measuring of frequency and frequency oscillations in multiphase power systems," IEEE Trans. Power Del., vol. 4, no. 1, pp. 95-102, Jan. 1989.

[13] E. Clarke, Circuit Analysis of A.C. Power Systems. New York: Wiley, 1943.

[14] M. Akke, "Frequency estimation by demodulation of two complex signals," IEEE Trans. Power Del., vol. 12, no. 1, pp. 157-163, Jan. 1997.

[15] A. Cataliotti, V. Cosentino, and S. Nuccio, "A phase-locked loop for the synchronization of power quality instruments in the presence of stationary and transient disturbances," IEEE Trans. Instrum. Meas., vol. 56, no. 6 , pp. 2232-2239, Dec. 2007.

[16] A. K. Pradhan, A. Routray, and A. Basak, "Power system frequency estimation using least mean square technique," IEEE Trans. Power Del., vol. 20, no. 3, pp. 1812-1816, Jul. 2005.

[17] B. Subudhi, P. K. Ray, S. R. Mohanty, and A. M. Panda, "A comparative study on different power system frequency estimation techniques," Int. J. Autom. Control, vol. 3, no. 2/3, pp. 202-215, May 2009.

[18] P. K. Dash, A. K. Pradhan, and G. Panda, "Frequency estimation of distorted power system signals using extended complex Kalman filter," IEEE Trans. Power Del., vol. 14, no. 3, pp. 761-766, Jul. 1999.

[19] M. M. Canteli, A. O. Fernandez, L. I. Eguiluz, and C. R. Estebanez, "Three-phase adaptive frequency measurement based on Clarke's transformation," IEEE Trans. Power Del., vol. 21, no. 3, pp. 1101-1105, Jul. 2006.

[20] D. Beeman, Industrial Power System Handbook. New York: McGraw-Hill, 1955.

[21] H. S. Song and K. Nam, "Instantaneous phase-angle estimation algorithm under unbalanced voltage sag conditions," Proc. Inst. Elect. Eng.-Gen. Transm. Distrib., vol. 147, no. 6, pp. 409-415, Nov. 2000.

[22] P. Rodriguez, J. Pou, J. Bergas, J. I. Candela, R. P. Burgos, and D. Boroyevich, "Decoupled double synchronous reference frame PLL for power converter control," IEEE Trans. Power Electron., vol. 22, no. 2, pp. 584-592, Mar. 2007.

[23] M. Mojiri, D. Yazdani, and A. Bakhshai, "Robust adaptive frequency estimation of three-phase power system," IEEE Trans. Instrum. Meas., vol. 59, no. 7, pp. 1793-1802, Jul. 2010.

[24] B. Picinbono and P. Chevalier, "Widely linear estimation with complex data," IEEE Trans. Signal Process., vol. 43, no. 8, pp. 2030-2033, Aug. 1995.

[25] P. J. Schreier and L. L. Scharf, "Second-order analysis of improper complex random vectors and process," IEEE Trans. Signal Process., vol. 51, no. 3, pp. 714-725, Mar. 2003.

[26] D. P. Mandic and S. L. Goh, Complex Valued Nonlinear Adaptive Filters: Noncircularity, Widely Linear and Neural Models. Hoboken, NJ: Wiley, 2009.

[27] S. Javidi, S. L. Goh, M. Pedzisz, and D. P. Mandic, "The augmented complex least mean square algorithm with application to adaptive prediction problems," in Proc. 1st IARP Workshop Cogn. Inform. Process., 2008, pp. 54-57.

[28] B. Picinbono, "On circularity," IEEE Trans. Signal Process., vol. 42, no. 12, pp. 3473-3482, Dec. 1994.
[29] B. Widrow, J. McCool, and M. Ball, "The complex LMS algorithm," Proc. IEEE, vol. 63, no. 4, pp. 719-720, Apr. 1974.

[30] S. L. Goh and D. P. Mandic, "An augmented extended Kalman filter algorithm for complex-valued recurrent neural networks," Neural Comput., vol. 19, no. 4, pp. 1039-1055, Apr. 2007.

[31] Y. Xia, B. Jelfs, M. M. V. Hulle, J. C. Príncipe, and D. P. Mandic, "An augmented echo state network for nonlinear adaptive filtering of complex noncircular signals," IEEE Trans. Neural Netw., vol. 22, no. 1, pp. 74-83, Jan. 2011.

[32] P. J. Schreier, "Bounds on the degree of impropriety of complex random vectors," IEEE Signal Process. Lett., vol. 15, pp. 190-193, 2008.

[33] D. C. Rife and R. R. Boorstyn, "Single-tone parameter estimation from discrete-time observations," IEEE Trans. Inf. Theory, vol. IT-20, no. 5, pp. 591-598, Sep. 1974.

[34] S. C. Douglas and D. P. Mandic, "Performance analysis of the conventional complex LMS and augmented complex LMS algorithms," in Proc. IEEE Int. Conf. Acoust., Speech Signal Process., 2010, pp. 3794-3797.

[35] D. P. Mandic, Y. Xia, and S. C. Douglas, "Steady state analysis of the conventional CLMS and augmented CLMS algorithms for noncircular complex signals," in Proc. Asilomar Conf. Signals, Syst. Comput., 2010, pp. 1635-1639.

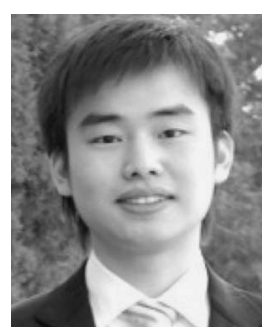

Yili Xia (S'09) received the B.Eng. degree in information engineering from Southeast University, Nanjing, China, in 2006, and the M.Sc. (with distinction) degree in communications and signal processing from the Department of Electrical and Electronic Engineering, Imperial College London, London, U.K., in 2007, where he is currently working toward the Ph.D. degree.

$\mathrm{He}$ is currently a Research Assistant with the Department of Electrical and Electronic Engineering, Imperial College London. His research interests include complex-valued linear and nonlinear adaptive filters and their applications on power systems.

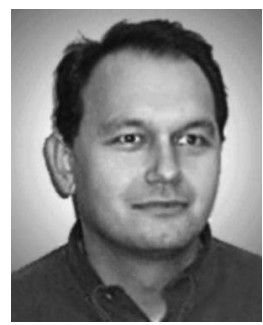

Danilo P. Mandic (M'99-SM'03) received the $\mathrm{Ph} . \mathrm{D}$. degree in nonlinear adaptive signal processing from Imperial College London, London, U.K., in 1999 .

$\mathrm{He}$ is a Reader in Signal Processing at Imperial College London, London, U.K. He has been working in the area of nonlinear adaptive signal processing and nonlinear dynamics. His publication record includes two research monographs titled Recurrent Neural Networks for Prediction: Learning Algorithms, Architectures and Stability (first edition, August 2001) and Complex Valued Nonlinear Adaptive Filters: Noncircularity, Widely Linear and Neural Models (first edition, Wiley, April 2009), an edited book titled Signal Processing Techniques for Knowledge Extraction and Information Fusion (Springer, 2008), and more than 200 publications on signal and image processing. He has been a Guest Professor at for Katholieke Universiteit Leuven, Leuven, Belgium, at Tokyo University of Agriculture \& Technology, Tokyo, Japan, and at Westminster University, London, and a Frontier Researcher in RIKEN Japan.

Dr. Mandic is a member of the London Mathematical Society. He is a member of the IEEE Technical Committee on Machine Learning for Signal Processing and an Associate Editor for the IEEE TRANSACTIONS ON CIRCUITS AND Systems II, the IEEE TRANSACTIONS ON Signal Processing, the IEEE TRANSACTIONS ON NEURAL NETWORKS, and the International Journal of Mathematical Modeling and Algorithms. He has produced award winning papers and products resulting from his collaboration with industry. 